\title{
Mit Social Media Lernprozesse analysieren und Problemfelder aufdecken
}

\author{
Christian Fischer \\ University of Tübingen
}

Abstract: Tausende Lehrkräfte tauschen sich täglich in den sozialen Netzwerken aus. Insbesondere Twitter ist ein Treffpunkt - nicht nur zum persönlichen Plausch, sondern auch für den professionellen Austausch mit anderen Lehrkräften über Schulbezirks- und Bundeslandgrenzen hinweg. Sie teilen Unterrichtsmaterialien und Links zu Informationsquellen, besprechen curriculare Veränderungen und Prüfungen und organisieren Chats, in denen sie fachliche Inhalte diskutieren oder einander emotional unterstützen. Dieser Austausch kann als eine Form der informellen Weiterbildung angesehen werden.

\section{Ist Lehrkräftefortbildung über Twitter effektiv?}

Getwittert wird vor allem in hashtag-gebundenen Gruppen, zum Beispiel im \#twitterlehrerzimmer. Mindestens 10.000 Tweets wurden darin im Jahr 2020 jeden Monat abgesetzt (Fütterer et al., 2021). Neben dieser allgemeinen Gruppe gibt es auch bundeslandspezifische, fachspezifische und themenspezifische Gruppen auf Twitter, in denen sich Lehrkräfte gezielter miteinander austauschen können.

Was die Lehrkräfte auf Twitter schätzen, sind vor allem die einfache Bedienung, die kostenlose Verfügbarkeit, die vielen Möglichkeiten zur Personalisierung und der geringe Aufwand, um aktiv oder passiv teilzunehmen. Im Gegensatz zu traditionellen Fortbildungen, die oftmals als Präsenzworkshops zu einem vorgegebenen Thema an einem einzigen Tag stattfinden, können Lehrkräfte in ihren Twitter-Gruppen räumlich und zeitlich flexibel, schnell und 
unkompliziert praxisnahe Ratschläge erhalten (Fischer, Fishman, \& Schoenebeck, 2019), wenn beispielsweise ein Physikexperiment nicht funktioniert.

Wir haben Online-Communities in den sozialen Netzwerken für Lehrkräfte naturwissenschaftlicher Fächer in den USA untersucht und konnten zeigen, dass sich die Leistung von Schülerinnen und Schülern verbesserte, wenn ihre Lehrkräfte sich in sozialen Netzwerken austauschten (Frumin et al., 2018).

\section{Chancen, Probleme schneller zu erkennen}

In weiteren Studien, die in Kooperation mit Kolleginnen und Kollegen aus den USA durchgeführt wurden, konnten wir belegen, wie Lehrkräfte mit anderen Akteuren im Bildungsbereich, beispielsweise der Wissenschaft oder der Politik, auf Twitter im Zusammenhang mit verschiedenen Bildungsreformen kommunizierten (Rosenberg et al., 2020a). Diese Vernetzung und Informationsfluss zwischen den unterschiedlichen Ebenen im Bildungsbereich ist eine große Chance, um beispielsweise Schwierigkeiten bei der Umsetzung von Reformen schnell erkennen und gegebenenfalls schnell nachsteuern zu können. Ferner haben wir herausgefunden, dass mithilfe der Stimmungen in den Tweets Rückschlüsse auf den Erfolg von Bildungsreformen gezogen werden können (Rosenberg et al., 2020b). Diese Information könnte beispielsweise in der Lehrkräftefortbildung nützlich sein, um Fortbildungen insbesondere in den Bereichen zu planen und anzubieten, in denen Lehrkräfte Schwierigkeiten sehen.

\section{Neue Möglichkeiten, Interaktion und Lernprozesse zu analysieren}

Für die empirische Bildungsforschung bietet Twitter in Verbindung mit den Analysewerkzeugen aus der Computerlinguistik neue, innovative Wege zur Erforschung von Lehrkräftefortbildungen. Im Vergleich zu traditionellen Fortbildungsformaten, die oftmals nur an Ort und Stelle mit Observationen oder retrospektiv mit Fragebögen oder Interviews erforscht 
werden können - und daher oftmals auch sehr kostspielig sind - bieten digitale Plattformen die Möglichkeit Handlung-, Interaktions-, und mögliche Lernprozesse auch noch lange nach der Durchführung nachvollziehen zu können.

Durch die Interaktionen von Nutzern mit den Plattformen werden automatisch große Mengen an Daten generiert, die nach Standards des „Educational Data Mining“ Forschungsfeldes in zwei Datenformate unterteilt werden können (Fischer et al., 2020): Zum einen entstehen „micro-level“ Logdaten, das heißt jedem abgesendeten Tweet wird automatisch eine Identifikationsnummer mit Zeitstempel zugewiesen, wobei auch Interaktionsmuster mit anderen Twitter-Usern wie Retweets oder Likes gespeichert werden. Mittels der Logdaten haben wir beispielsweise die Hierarchie und Kollaborationsstrukturen von Usern innerhalb der Gruppen betrachtet (Fischer et al., 2019). Zum anderen entstehen „meso-level“ Textdaten aus der Gesamtheit aller Tweets. Diese bieten neue Möglichkeiten, die Interaktions- und Lernprozesse in Twitter-Communities feingliedrig zu analysieren. Mit den Textdaten haben wir sowohl den Inhalt als auch die Emotionen innerhalb einzelner Tweets oder Konversationen analysiert (Rosenberg et al., 2020b). Vielversprechende Anwendungen könnten außerdem Beobachtungswerkzeuge sein, die automatisiert die „Public Opinion“ von Social Media Plattformen abbilden. Mit ihnen könnte die Bildungspolitik Hinweise zu möglichen Problemfeldern erhalten, die zum Beispiel im Zuge von Bildungsreformen entstehen.

\section{References}

Fischer, C., Fishman, B., \& Schoenebeck, S. (2019). New contexts for professional learning: Analyzing high school science teachers' engagement on Twitter. AERA Open, 5(4), 1-20. https://doi.org/10.1177/2332858419894252 
Fütterer, T., Hoch, E., Stürmer, K., Lachner, A., Fischer, C., \& Scheiter, K. (2021). Was bewegt Lehrpersonen während der Schulschließungen? - Eine Analyse der Kommunikation im Twitter-Lehrerzimmer über Chancen und Herausforderungen digitalen Unterrichts. Zeitschrift für Erziehungswissenschaft, 24, 443-477. https://doi.org/10.1007/s11618-02101013-8

Fischer, C., Pardos, Z., Baker, R. S., Williams., J. J., Smyth, P., Yu, R., Slater, S., Baker, R., \& Warschauer, M. (2020). Mining big data in education: Affordances and challenges. Review of Research in Education, 44(1), 130-160. https://doi.org/10.3102/0091732X20903304

Frumin, K., Dede, C., Fischer, C., Fishman, B., Eisenkraft, A., Foster, B., Levy, A., Lawrenz, F., \& McCoy, A. (2018). Adapting to large-scale changes in Advanced Placement biology, chemistry, and physics: The impact of online teacher communities. International Journal of Science Education, 40(4), 397-420. https:/doi.org/10.1080/09500693.2018.1424962

Rosenberg, J. M., Reid, J. W., Dyer, E., Koehler, M., Fischer, C., \& McKenna, T. J. (2020a). Idle Chatter or Compelling Conversation? The Potential of the Social Media-based \#NGSSchat Network for Supporting Science Education Reform Efforts. Journal of Research in Science Teaching, 57(9), 1322-1355. https://doi.org/10.1002/tea.21660

Rosenberg, J., Borchers, C., Dyer, E., Anderson, D. \& Fischer, C. (2020b). Advancing new methods for understanding public sentiment about educational reforms: The case of Twitter and the Next Generation Science Standards. OSF Preprints. https://doi.org/10.31219/osf.io/xymsd 
Anmerkung: Eine Version dieses Beitrages erscheint im 2019-2020 Jahresbericht vom Hector-Institut für Empirische Bildungsforschung der Universität Tübingen. 